\title{
Mathematical Model on the Effects of Global Climate Change and Decreasing Forest Cover on Seasonal Rainfall
}

\author{
Genet Mekonnen Assefa (Msc) \\ Debark University College of Natural and Computational Sciences, Department of Mathematics
}

\begin{abstract}
This study involves the study of the long-term behaviors of rainfall as it is affected by changes to forest area and the rise in global temperature. Global temperature and forest cover are considered annually while the amount of rainfall are considered seasonally to best capture the effects of severe weather hazards such as drought and puberty. A differential equation model was developed and verified using the mean global temperature annually, forest area, the daily amounts of rainfall. The rise in global temperature as well as the decline in forest area can be, as shown in the seminar, represented by logistic equations. Rainfall is, however, represented as a periodic function; hence, second order differential equation, of which the solution is periodic, is used to represent the rate of change in the amount of rainfall. In addition, by correlation analysis, the predator-prey terms of forest, global temperature and rainfall are presented in the models.
\end{abstract}

DOI: $10.7176 / \mathrm{MTM} / 9-1-03$

\section{Chapter 1 Introduction}

Human beings have become a component in the earth's system, driving and accelerating global warming through the rapid release of green house gases (GHGs) into the atmosphere. Climate change is a major concern affecting all organisms. Over the past 30 years, global surface temperature has been rising approximately 0:20C per decade. Severe weather conditions such as drought and flood have been known to result from high global temperature. The amount of greenhouse gases (especially carbon dioxide) in the atmosphere depends inversely on the amount of forest area. Today, forest cover has decreased from the past due to timber demands for building and manufacturing, [?] and to land usage for agriculture.

Analysis of climate change and its extreme is becoming more important as it clearly affects the human society and are essential for exploration of ecological and societal changes. Climate change is an irreversible consequence of the global warming phenomenon. Global warming, or the greenhouse effect, has been brought about by an increase in greenhouse gases (GHG) in the atmosphere. Climate change will impact on bio-physical systems and ultimately will have consequences to human well being. Understanding climate change is fundamental to prepare and to cope with future risk. Climate change is not uniform over space and time and its impact on bio-physical system varies from place to place. Therefore, it is necessary to understand climate change at the local scale and aim to get site-specific information. Climate change, which is induced by global warming, has become a global concern because it has the potential to impact many systems and sectors which would threaten human well being.

The El Nino phenomenon has played an important role on the behavior of rainfall in different areas of the world; it is also a major cause of extreme weathers in many regions of the world [?]. Changes in global temperature, therefore, lead to variations in the amount of rainfall in different areas of the world, an amount which depends on the level of forest transpiration. Deforestation is the single largest cause of forest area diminution, even compared to natural disasters such as wildlife. The amount of rainfall is an important indicator for severe weather conditions such as drought and flood.

Many mathematical models in ecology have been developed to describe the relationship between carbon dioxide and global warming, forest and carbon dioxide or greenhouse gases, climate change and extreme weathers, species (or population) survival and pollution, i.e., industrialization, species (or population) survival and forestry or biomass resources and industrialization, population, and pollution. There is also a study to control the amount of pollution in the environment in order to restrain the global carrying capacity of population. In another study, 
the dynamic of the relationship between biomass (which can be viewed as forestry resources), industrialization, population, pollution, and pollution released by the biomass resources (which obviously includes carbon dioxide) was written in the form of a system of differential equations. Furthermore, there is a mathematical model related to the competition among rain forest species using the Lotka-Volterra predator-prey model [?]. However, a mathematical model that simultaneously represents the relationship between global temperature, amount of forest area and amount of rainfall has never been presented.

\subsection{Statement of the Problem}

Changes in global climate would significantly affect human health, natural aquatic and terrestrial ecosystems, and agricultural ecosystems. World-wide attention recently has turned to these issues and scientists from many disciplines and many countries are working to assess the potential magnitude and direction of the changes and the risks to the biota. This study is intended to answer the following basic questions:

1. Can we formulate a mathematical model that describes the relationship between global temperature dynamics, forest area and amounts of rainfall?

2. What are the impacts of the rise in global temperature and decline in forest area on stability of seasonal rainfall?

3. Is there the interaction between global temperature, forest area and rainfall?

\subsection{Objective of the Study}

\subsubsection{General Objective:}

The general objective of this study is to develop a mathematical model of the effects of global climate change and decreasing in forest cover on seasonal rainfall.

\subsubsection{Specific Objectives:}

The principal objectives of this study are:

$>$ To develop a mathematical model that describe the relationship between global climate change and decreasing forest area on seasonal rainfall

$>$ To study the effects of the rise in global temperature and decline of forest area on the stability of seasonal rainfall

$>$ To know the interactions between global temperature, forest area and rainfall

\subsection{Significance and Beneficiaries}

This study provides reliable information on how we can use the mathematical modeling to know the effects of global temperature and decreasing forest cover on seasonal rainfall. The outcome of the study benefits building and running of a model is a process by which theory and observations are mathematically evaluated, codified and integrated. Also, initiate other researchers to identify and then assimilate observational measurements that are initially incomplete.

\section{Chapter 2 Literature Review}

The first global models (models of world dynamics) were developed by J.W. Forrester and D.H. Meadows in the 1970's. They produced mostly qualitative results and represented first systematic attempts to analyze global trends. These models evolved into a more quantitative integrated assessment (IA) approach in global modeling. The Intergovernmental Panel on Climate Change (IPCC) was jointly established by the World Meteorological Organization and the United Nations Environment Programed in 1988, in order to:

(i) Assess available scientific information on climate change,

(ii) Assess the environmental and socio-economic impacts of climate change, and

(iii) Formulate response strategies.

Also, James Walsh and Richard McGehee model the climate dynamically. In James Walsh, (1991), proposed a mathematical model climate dynamics to extend the work of Richard McGehee, (1969). Global climate is determined by the radiation balance of the planet. The Earth warms through the absorption of incoming solar radiation (or insolation). For the global climate model, the variable of interest is the annual global mean surface 
temperature $\mathrm{T}=\mathrm{T}(\mathrm{t})$. The annual global mean insolation is known as the solar constant $\mathrm{Q}$. The incoming energy absorbed by the Earth is then modeled by the term $\mathrm{Q}(1-\alpha)$, where $\alpha$ is the average global insolation reflected back into space. The loss of energy is modeled by a linear approximation A + BT, with parameters A and B. and finally its model equation for the annual global mean temperature is then given by

$$
R \frac{d T}{d t}=Q(1-\alpha)-(A+B T)
$$

where the left-hand side of this equation represents the change in energy stored in the Earth's surface. The parameter $\mathrm{R}$ is the heat capacity of the Earth's surface.

Bampfylde et al., (2005) introduces a mathematical model related to the competition among rain forest species using the Lotka-Volterra predator-prey model. Dubey and Narayanan, (2010) models a dynamic of the relationship between biomass, industrialization, population, pollution, and pollution released by the biomass resources was written in the form of a system of differential equations.

Md. Hamidul Islam and Md. Abdus Salam et al.,(2011) uses a mathematical model to compute the crucial roles of water vapor in global warming by expressing the temperature $\mathrm{T}$ as a function of carbon dioxide $\mathrm{C}$, that is $\mathrm{T}(\mathrm{t})$ $=\mathrm{f}(\mathrm{C})$. The developed differential equation is,

$$
\frac{d T}{d t}=\frac{d T}{d C} \frac{d C}{d t}=k \frac{d C}{d t}
$$

The term $\left(\frac{d T}{d C}=k\right)$ denotes the change of temperature per unit change in $\mathrm{CO} 2$ concentration in the atmosphere. This model of global warming is without the effect of water vapor.

However, a mathematical model that simultaneously represents the relationship between global temperature, amount of forest area and amount of rainfall has never been presented.

\section{Chapter 2 Mathematical Preliminary}

In this chapter, we state some theorems and give the definitions of terminologies which are most crucial for the seminar as an input.

\subsection{Existence and Uniqueness Theorem}

Before one spends much time attempting to solve a given differential equation, it is wise to know that solutions actually exist. We may also want to know whether the solution is uniquely satisfy the equation with the given initial condition. We consider here the autonomous system in $\mathrm{R}^{\mathrm{n}}$. i.e., a collection of equations that do not explicitly contain the independent variable. More generally, autonomous systems have the form

$x^{\prime}=f(x)$
Where $x^{\prime}=\left[\begin{array}{c}x_{1} \\ x_{2} \\ \vdots \\ x_{n}\end{array}\right]$ and $f=\left[\begin{array}{c}f_{1}\left(x_{1}, x_{2}, \ldots x_{n}\right) \\ f_{2}\left(x_{1}, x_{2}, \ldots x_{n}\right) \\ \vdots \\ . \\ f_{n}\left(x_{1}, x_{2}, \ldots x_{n}\right)\end{array}\right]$

Theorem 3.1.1. (Existence and Uniqueness Theorem): Assume D is an open subset of $R \times R_{n}, f: D \rightarrow R^{n}$ is a continuous. Then for each $t_{0} \in R$ and $x_{0} \in R^{n}$, the IVP

$x^{\prime}=f(t, x), x\left(t_{0}\right)=x_{0}$

Has a solution $\mathrm{x}$. if in addition, $f$ has continuous first order partial derivatives with respect to $x_{1}, x_{2}, \ldots x_{n}$ on $R_{n}$, then the above IVP has a unique solution. The following known lemma is useful for the prove of this theorem.

Lemma 2.1.2. Assume that $\mathrm{f}$ is continuous $\mathrm{n}$-dimensional vector function on the rectangle $R:=\left\{(t, x): t_{0} \leq t \leq t_{0}+a,\left\|x-x_{0}\right\| \leq b\right\}$ and assume that $f(t, x)$ satisfies a uniform Lipchitz condition with respect to $x$ on $\mathrm{R}$.

Let $M:=\max \{\|f(t, x)\|:(t, x) \in R\}$ and $\alpha:=\min \left\{a, \frac{b}{M}\right\}$ Then the IVP has a unique solution $x$ on $\left[t_{0}, t_{0}+\right.$ $\alpha]$

Proof.(i) Existence

Given $f(t, x)$ as well as $\left(t_{0}, x_{0}\right)$, demarcate a neighborhood $\mathrm{N}$ around the central point and use it to define the constants $a^{\prime}, b^{\prime}$ Picard mapping $p$ and $h_{0}$ a point of contraction mapping A.

The function $g: R \times R^{n} \rightarrow R^{n}$ given by 
is therefore always well defined in neighborhood of $\left(t_{0}, x_{0}\right)$. Applying the Picard mapping,

$$
g(t, x)=x+h_{0}(t, x)
$$

$$
(P g)(t, x)=x+\left(A h_{0}\right)(t, x)=x+h_{0}(t . x)=g(t, x)
$$

Which proves that, $g$ is a solution of a differential equation which satisfies the initial condition $g\left(t_{0}, x\right)=x$ as long as $t$ is in a neighborhood of the point $t_{0}$ defined by $\left|t-t_{0}\right| \leq a^{\prime}$ and $x$ is any point such that $\left|x-x_{0}\right| \leq b^{\prime}$.

(ii) Uniqueness

Let $\left(t_{0}, x_{0}\right) \in D$; then there are positive numbers $a$ and $b$ such that the rectangle

$R:=\left\{(t, x):\left|t-t_{0}\right| \leq a,\left\|x-x_{0}\right\| 1 \leq b\right\} \subset D$

By the hypothesis of the theorem, $f(t, x)$ satisfies a Lipchitz condition with respect to $\mathrm{x}$ on $\mathrm{R}$ with Lipchitz constant. $\quad K:=\max \left\{\left\|D_{x} f(t, x)\right\|:(t, x) \in R\right\}$

Where $\|$.$\| is a matrix norm corresponding to the norm \|.\|_{1}$.

Let $M:=\max \{\|f(t, x)\|:(t, x) \in R\}$ and let $\alpha:=\min \left\{a, \frac{b}{m}\right\}$ then by Picard-Lindale theorem, the IVP (2.1.2) has a unique solution on $\left[t_{0}-a, t_{0}+a\right]$

Bounded function: Let $\mathrm{f}$ be a real valued function defined on a domain $D$. The function $f$ is said to be bounded on $D$ if and only if there is a positive number $M$ such that $f(x, y) \leq M$ for all $(x, y) \in D$.

Definition 2.1.1. A solution $\varphi: t \rightarrow \varphi(t)$ or $x^{\prime}=f(x)$ is called periodic with period $T$ or $T$-periodic if $\varphi(t+$ $T)=\varphi(t)$ for all t. If, moreover, $\varphi(t+\tau) \neq \varphi(t)$ for any $\tau \in(0, T)$ then $T$ is called the minimal period.

\section{Linearization}

In this section we will see the behavior of solutions of non-linear system

$$
x^{\prime}=f(x)
$$

Near an equilibrium $\mathrm{x} 0$ by relating to the (2.1.3) directly to a linearized system

$$
x^{\prime}=D f\left(x_{0}\right)\left(x-x_{0}\right)
$$

about that same equilibrium, where $D f\left(x_{0}\right)$ is the $n \times n$ matrix of partial derivatives of $f . D f=\left(\partial f_{i} / \partial x_{j}\right)^{n}$ evaluated at $x_{0}$. A linear system of first order ordinary differential equations is defined by

$$
x^{\prime}(t)=A(t) x(t)+B(t)
$$

where the unknown $x$ is a map on an interval of $\mathrm{R}$, say $\Gamma$, taking values in a normed vector space $E$ over a field $K$, defined differentiable on a sub interval of $\Gamma$. The name linear for system the above equation is an abuse of language. The associated linear system of (2.1.4) is

$A(t) x(t)$

$x^{\prime}(t)=$

Another way to distinguish systems (2.1.4) and (2.1.5) is to refer to the former as a non -homogeneous linear system and the latter as an homogeneous linear system.

\subsection{Equilibrium Solutions and Stability}

Stability: The quality of being steady and not changing or being disturbed in any way. Consider the general population equation.

$$
\frac{d x}{d t}=(\beta-\delta) x
$$

Where $\beta$ and $\delta$ are the birth and death rates, respectively, in births or deaths per individual per unit of time. The question of whether a population $x(t)$ is bounded or unbounded as $t \rightarrow \infty$ is of evident interest. In many situations like the logistic populations, the birth and death rates are known functions of $x$. Then equation (2.2.1) takes the form

$$
\frac{d x}{d t}=f(x)
$$

This is an autonomous first order ordinary differential equation one in which the independent variable $t$ does not appear explicitly. The solutions of the equation $f(x)=0$ play an important role and are called critical points of the autonomous differential equations (2.2.2). If $x=c$ is a critical point of (2.2.2), then the differential equation has the constant solution $x(t)=c$. A constant solution of a differential equation is sometimes called an equilibrium solution (one may think of a population that remains constant because it is in "equilibrium" with its environment). Thus the critical point If $x=c$, a number, corresponds to the equilibrium solution If $x(t)=c$, a constant valued function.

\section{Stability of critical points}


A critical point $x=c$ of an autonomous first order ordinary differential equation is said to be stable provided that, if the initial value $x_{0}$ is sufficiently close to $\mathrm{c}$, then $x(t)$ remains close to $\mathrm{c}$ for all $c>0$. More precisely, the critical point $\mathrm{c}$ is stable if, for each $\varepsilon>0$, there exists $\delta>0$ such that

$$
\left|x_{0}-c\right|<\delta
$$

implies that $|x(t)-c|<\delta$ for all $\mathrm{t}>0$. The critical point $\mathrm{x}=\mathrm{c}$ is unstable if it is not stable.

Theorem 2.2.1 (Stability Theorem) Assume A is an $n \times n$ constant matrix.

(i) If $A$ has an eigenvalue with positive real part, then the trivial solution is unstable on $[0, \infty)$.

(ii) If all the eigenvalues of $\mathrm{A}$ with zero real parts are simple (multiplicity one) and all other eigenvalues of $\mathrm{A}$ have negative real parts, then the trivial solution is stable on $[0, \infty)$.

(iii) If all the eigenvalues of $\mathrm{A}$ have negative real parts, then the trivial solution of $x=A x$ is globally asymptotically stable on $[0, \infty)$.

Definition 2.2.1 let $x_{0}$ be an equilibrium point for $x_{0}=f(x)$. A continuously differentiable function $\mathrm{V}$ defined on an open set $U \in R^{n}$ with $x_{0} \in U$ is called a Liapunov function for $x^{\prime}=f(x)$ on $\mathrm{U}$ provided $V\left(x_{0}\right)>0$; $V(x)>0$ for $x \neq x_{0}, x \in U$ and

$$
\operatorname{gradV}(x) . f(x) \leq 0
$$

for $x \in U$. If the inequality (2.2.3) is strict for $x \in U: x \neq x_{0}$, then $\mathrm{V}$ is called a strict Liapunov function for $x_{0} \in$ $f(x)$ on $\mathrm{U}$.

Note that (2.2.3) implies that if $x \in U$, then

$\frac{d}{d t} V(\varnothing(t, x))=\operatorname{grad} V(\varnothing(t, x)) \cdot f(\varnothing(t, x)) \leq 0$,

As long as $\emptyset(t, x)$ remains in $U$, so $V$ is decreasing along orbits as long as they stay in $U$.

Here is Liapunov's stability theorem:

Theorem 2.2.2: (Liapunov's Stability Theorem) If V is a Liapunov function for $x^{\prime}=f(x)$ on an open set $U$ containing an equilibrium point $x_{0}$, then $x_{0}$ is stable. If $\mathrm{V}$ is a strict Liapunov function, then $x_{0}$ is asymptotically stable [2].

Proof: Assume V is a Liapunov function for (3.1.1) on an open set $\mathrm{U}$ containing an equilibrium point $x_{0}$. Pick r $>$ 0 sufficiently small so that $B\left(x_{0}, r\right) \subset U$ and define

$$
\text { Now } \quad W=\left\{x: V(x)<\frac{m}{2}\right\} \cap B\left(x_{0}, r\right)
$$

Is open \& contain $x_{0}$, choose $x>0$ so that $B\left(x_{0}\right), s \subset W$, for $x \in B\left(x_{0}\right), \mathrm{s}$,

$$
V(\varnothing(t, x)) \leq \frac{m}{2}
$$

as long as $\emptyset(t, x)$ remains in $W$ since $V(\varnothing(t, x))$ is decreasing. Thus $\emptyset(t, x)$ cannot intersect the boundary of $B\left(x_{0}, r\right)$ for $t \geq 0$, so $\emptyset(t, x)$ remains in $B\left(x_{0}, r\right)$ for $t \geq 0$, and $x_{0}$ is stable.

Now suppose $V$ is a strict Liapunov function, but $x_{0}$ is not asymptotically stable. Then there is an $x \in B\left(x_{0}, s\right)$ so that $\emptyset(t, x)$ does not go to $x_{0}$ as $t \rightarrow \infty$ t. Since the orbit is bounded, there is an $x_{1} \neq x_{0}$, and a sequence $x_{k} \rightarrow \infty$ so that $\varnothing\left(t_{k}, x\right) \rightarrow x_{1}$ as $k \rightarrow \infty$.

Note that by semi-group property for orbits

$$
\begin{gathered}
\emptyset\left(t_{k}+1, x\right)=\emptyset\left(1, \emptyset\left(t_{k}, x\right)\right) \\
\text { As } k \rightarrow \infty \quad V\left(\varnothing\left(t_{k}+1, x\right)\right)=V\left(\varnothing\left(1, \emptyset\left(t_{k}, x\right)\right)\right) \rightarrow V\left(\varnothing\left(1, x_{1}\right)\right)<V\left(x_{1}\right)
\end{gathered}
$$

So there is an integer $N$ for which $V\left(\varnothing\left(t_{N}+1, x\right)\right)<V\left(x_{1}\right)$

Choose $k$ so that $t_{k}>t_{N}+1$ then

$$
V\left(x_{1}\right) \leq V\left(\varnothing\left(t_{k}, x\right)\right)<V\left(\varnothing\left(t_{N}+1, x\right)\right)
$$

a contradiction. We conclude that $\mathrm{x} 0$ is asymptotically stable.

\subsection{Logistic Equation}

A differential equation of the form

$$
\frac{d P}{d t}=a P-b P^{2}
$$

That models a population $P(t)$ with births (per unit time) proportional to $P$ and deaths proportional to $P^{2}$, where the coefficients $a$ and $b$ are positive is called the Logistic Equation. The logistic equation $\frac{d P}{d t}=a P-b P^{2}, a>$ $0, b>0$ is an autonomous first order differential equation. The finite limiting population is the characteristic of logistic populations. 
The solution of the logistic initial value problem

Is

$$
P(t)=\frac{\frac{d P}{d t}=a P-b P^{2}, P\left(t_{0}\right)=p_{0}}{b P_{0}+\left(a-b P_{0}\right) e^{-a\left(t-t_{0}\right)}}
$$

One method of solving (2.3.2) is separation of variables.

$$
\begin{gathered}
\frac{d P}{d t}=a P-b P^{2} \\
\Rightarrow \int_{P_{0}} \frac{d P}{P(a-b P)}=\int_{t_{0}}^{t} d t
\end{gathered}
$$

But decomposing the left side of $\frac{d P}{P(a-b P)}=d t$ in to partial fractions gives

$$
\frac{1}{P(a-b P)}=\frac{1 / a}{P}+\frac{b / a}{a-b P}
$$

$$
\begin{gathered}
=\frac{1}{a}\left(\frac{1}{p}-\frac{-b}{a-b p}\right) \\
\therefore \frac{1}{a} \int_{P_{0}}^{P} \frac{d P}{P}-\frac{1}{a} \int_{P_{0}}^{P} \frac{-b d P}{a-b P}=\int_{t_{0}}^{t} d t
\end{gathered}
$$

Solving we have

We can now solve for $\mathrm{P}(\mathrm{t})$

$$
\begin{gathered}
{\left[\frac{1}{a} \ln P-\frac{1}{a}(-b) \ln (a-b P)\left(\frac{1}{-b}\right)\right]_{P_{0}}^{P}=t-t_{0}} \\
\frac{1}{a} \ln \left(\frac{P}{P_{0}}\right)-\frac{1}{a} \ln \left(\frac{a-b P}{a-b P_{0}}\right)=t-t_{0} \\
\frac{\ln \left(P\left(a-b P_{0}\right)\right)}{P_{0}(a-b P)}=a\left(t-t_{0}\right) \\
\therefore P_{0}(a-b P) e^{a\left(t-t_{0}\right)}=P\left(a-b P_{0}\right)
\end{gathered}
$$

$$
\begin{gathered}
P_{0} e^{a\left(t-t_{0}\right)}=P b P_{0} e^{a\left(t-t_{0}\right)}+P\left(a-b p_{0}\right)=P\left[b P_{0} e^{a\left(t-t_{0}\right)}+\left(a-a P_{0}\right)\right] \\
\text { i.e } P(t)=\frac{a P_{0}}{b P_{0}+\left(a-b P_{0}\right) e^{-a\left(t-t_{0}\right)}}
\end{gathered}
$$

This represents the logistic function and it has an upper limit of

$$
\lim _{t \rightarrow \infty} P(t)=\frac{a P_{0}}{b P_{0}}=\frac{a}{b}
$$

Thus a population that satisfies the logistic equation does not grow without bound like a naturally growing population modeled by the exponential equation $P^{\prime}=k P$. Instead, it approaches the finite limiting population $\frac{a}{b}$, as $t \rightarrow \infty$.

The autonomous differential equation

$$
\frac{d x}{d t}=a x-b x^{2}-h
$$

(with a, b, and $h$ all positive) may be considered to describe a logistic population with harvesting. For instance, we might think of the population of fish in a lake from which $h$ fish per year are removed by fishing. We can rewrite eqn.(2.3.4) in the form

$$
\frac{d x}{d t}=k x(M-x)-h
$$

Which exhibits the limiting population $M$ in the case $h=0$ of no harvesting, where $k=b$ and $M=\frac{a}{b}$ are constants.

Predator-prey model: The classical mathematical model of a predator-prey model was developed in the 1920s by the Italian mathematician Vito Volterra (1860-1940) in order to analyze the cyclic variations observed in the shark and food fish populations in the Adriatic Sea. For this consider a predator-prey situation involving two species: one species the predators-feeds on the other species and the prey-which in turn feeds on some third food item readily available in the environment. A standard example is a population of foxes and rabbits in a 
woodland; the foxes (predators) eat rabbits (the prey), while the rabbits eat certain vegetation in the woodland [1].

\section{Chapter 3 Modeling the Effects of Global Climate Change}

In this chapter, we present model description and formulation. The mathematical theory of climate is a branch of the theory of climate, which investigates the behavior of the climate models solutions on the arbitrarily large time scales by the use of a collection of mathematical methods. This seminar relies on the hypothesis that changes in global temperature, forest area and amount of rainfall are related.

\subsection{The assumptions}

The following assumptions are made in order to construct the model:

(i) The behavior of global temperature is apparently increases exponentially. However, the global temperature cannot possibly increase forever. It should be bounded at some temperature level.

(ii) Today the forest area have been decreased exponentially from the past. Since the forest area cannot decrease below zero and cannot be larger than the maximum area of the studied region, be an example of a logistic decay.

(iii) The amount of rainfall is apparently seasonal and therefore periodic. The second order differential equation with periodic solution should be considered to capture the seasonal rainfall.

\subsection{Model Formulation and Analysis}

From the model description, assumptions and definition of variables and parameters in section (4.1), the formulated models are as follows: The dynamics of the global temperature are assumed by a logistic equation instead of an exponential equation. When the exponential growth behavior is unbounded, the logistic growth with bounded behavior should be considered instead. The relationship leading to the logistic equation form is $\frac{d T}{d t} \propto T$ and $T^{2}$. Therefore, we assume the equation $\frac{d T}{d t}$ to be

$$
\frac{d T}{d t}=a_{T}\left(1-\frac{T-m_{T}}{b_{T}}\right)\left(T-m_{T}\right)
$$

Eq. (3.2.1) can be rearranged in the form

$$
\frac{d T}{d t}=k_{1}+a_{1} T-b_{1} T^{2}
$$

Where $a_{1}=a_{T}\left(1+\frac{2 m_{T}}{b_{T}}\right), b_{1}=a_{T} /_{b_{T}}$ and $k_{1}=-a_{T} m_{T}\left(1+m_{T} /_{b_{T}}\right)$

The dynamics of forest area are also assumed by a logistic equation instead of an exponential equation. Similar to the previous one, the well-known relationship for the logistic equation is $\frac{d F}{d t} \propto F$ and $F^{2}$, then

$$
\frac{d F}{d t}=-a_{F}\left(1-\frac{F-m_{F}}{b_{F}}\right)\left(F-m_{F}\right)
$$

Eq. (3.2.2) can be rearranged in the form

$$
\frac{d F}{d t}=k_{2}-a_{2} F-b_{2} F^{2}
$$

Where $a_{2}=a_{F}\left(1+2 m_{F} / b_{F}\right), b_{2}=-a_{F} / b_{F}$ and $k_{2}=a_{F} m_{F}\left(1+m_{F} / b_{F}\right)$

The amount of periodic rainfall will be represented by the following second order differential equation

$$
\frac{d^{2} R}{d t^{2}}=-e_{s}^{2}(R-c)
$$

Since the other two variables can be represented by first order differential equations, this second order equation representing the behavior of rainfall will be reduced to two first order differential equations. Introducing an intermediate variable $S$ as the rate of change of rainfall, the amount of periodic rainfall will be represented by the following system of first order differential equations.

$$
\begin{aligned}
& \frac{d R}{d t}=S \\
& \frac{d S}{d t}=-e_{s}^{2}(R-c)
\end{aligned}
$$

\subsubsection{Stability analysis}

In this part of the seminar we present the stability of non-negative equilibrium in Equation (3.2.1)-(3.2.4).

Theorem 3.2.1 Equation (3.2.1) has stable equilibrium point.

Proof: Observe that when $\frac{d T}{d t}=0$ we have the equilibrium points $T=m_{T}$ and $T=m_{T}+b_{T}$

The exact solution of Eq. (3.2.1) is 
Where $A=\left(b_{T}\left(T_{0}-m_{T}\right)\right) /\left(b_{T}-T_{0}+m_{T}\right.$ and $T(0)=T_{0}$.

$$
T(t)=m_{T}+\frac{b_{T} A}{A+b_{T} e^{-a_{T} t}}
$$

We obtain $\lim _{t \rightarrow \infty} T(x)=m_{T}+b_{T}$ hence, $m_{T}+b_{T}$ is the stable equilibrium point and $m_{T}$ is the minimum global temperature and $b_{T}$ is the difference between two equilibrium points.

Theorem 3.2.2 Equation (3.2.2) has stable equilibrium point.

Proof: Similar to Eq. (3.2.1) and (3.2.2) has the equilibrium points at $F=m_{F}$ and $F=m_{F}+b_{F}$

The exact solution for Eq. (3.2.2) is

$$
F(t)=m_{F}+\frac{b_{F} B}{B+b_{F} e^{a_{F} t}}
$$

Where $B=\left(b_{F}\left(F_{0}-m_{F}\right)\right) /\left(b_{F}-F_{0}+m_{F}\right)$ and $F(0)=F_{0}$

We obtain $\lim _{t \rightarrow \infty} F(t)=m_{F}$ Hence, $m_{F}$ is the stable equilibrium point and also the minimum forest area.

Theorem 3.2.3 The system of equation (3.2.4) has a stable solution.

Proof: This equation can be written in the form

$$
\begin{gathered}
\left(\begin{array}{l}
R^{\prime} \\
S^{\prime}
\end{array}\right)=\left(\begin{array}{cc}
0 & 1 \\
-e_{s}^{2} & 0
\end{array}\right)\left(\begin{array}{l}
R \\
S
\end{array}\right)+\left(\begin{array}{c}
0 \\
c e_{s}^{2}
\end{array}\right) \\
R(t)=c+C_{1} \cos \left(e_{s} t\right)+C_{2} \sin \left(e_{s} t\right)
\end{gathered}
$$

Where $C_{1}=R_{0}-c, C_{2}=\frac{s_{0}}{e_{s}}, R(0)=R_{0}, S(0)=S_{0}$

The critical point is the solution to the system

Solving the system we obtain the critical point $(c, 0)$

$$
\left\{\begin{array}{c}
S=0 \\
-e_{S}^{2} R+c e_{S}^{2}=0
\end{array}\right.
$$

Now use the change of variable

$$
R=u+c, s=v
$$

to translate the critical point to the origin $(0,0)$

$$
\begin{gathered}
\frac{d R}{d t}=\frac{d u}{d t}=v \\
\frac{d s}{d t}=\frac{d v}{d t}=-e_{s}^{2}(u+c)+c e_{s}^{2}=-e_{s}^{2} u
\end{gathered}
$$

Then we get

$$
\begin{aligned}
& \frac{d u}{d t}=v \\
& \frac{d v}{d t}=-e_{s}^{2} u
\end{aligned}
$$

The characteristic equation of the coefficient matrix, say

Is $\operatorname{det}(A-\lambda I)=\lambda^{2}+e_{S}^{2}=0$

$$
A=\left(\begin{array}{cc}
0 & 1 \\
-e_{s}^{2} & 0
\end{array}\right)
$$

Solving we obtain $\lambda_{1,2}= \pm e_{s} i$. Since all the Eigen values of $\mathrm{A}$ have zero real parts by theorem 3.3.1 the above system of equation has a stable solution.

\subsection{Modified models with predator-prey terms}

The amount of forest area, amount of rainfall and global temperature are used to obtain the correlations between the rates of change of each variable, $\frac{d T}{d t}, \frac{d F}{d t}, \frac{d R}{d t}, \frac{d S}{d t}$ and the other terms including the cross products or the predator-prey terms. Note that under our preliminary hypothesis, the rates of change in forest area and rainfall are affected by the global temperature. On the other hand, there can be many other factors affecting the rate of global temperature change such as waste gases from industry or transportation, etc. The cross product terms between global temperature and others are not shown in the dynamics of the global temperature.

The modified models with predator-prey terms are

$$
\begin{gathered}
\frac{d T}{d t}=a_{T}\left(1-\frac{T-m_{T}}{b_{T}}\right)\left(T-m_{T}\right) \\
\frac{d F}{d t}=-a_{F}\left(1-\frac{m_{F}}{b_{f}}\right)\left(F-m_{F}\right)+e f R+\alpha_{F} F R+\beta_{F} T F-\pi_{F} T R \\
\frac{d R}{d t}=f_{R} S-\alpha_{R} F R-\pi_{R} T R+\gamma_{R} F S+\mu_{R} T S+\delta_{R} R S
\end{gathered}
$$




$$
\frac{d S}{d t}=-e_{S}^{2}(R-c)+\beta_{S} T F+\pi_{S} T R-\gamma_{S} F S-\mu_{S} T S-\delta_{S} R S
$$

The new parameter, $e f$, represents the rate of change in forest area caused by the amount of water (rainfall) absorption.

The terms FR in Eq. (3.3.1) represent the proportion of the amount of rainfall in the forest and FS is the proportion of the rainfall difference rate (or the rate of change in rainfall) in the forest. The coefficient $\alpha_{F}$, is the growth rate of the forest affected by the proportion of rainfall in the forest. The constant $\alpha_{R}$ is the rate of decrease that indicates some amount of rainfall remaining in the forest. The parameters, $\mathrm{R}$ and $\mathrm{S}$, are both the difference rates representing the changing behavior of the rain falling in the forest.

The TF terms in Eq. (3.3.1) indicate the proportion of the forest cover at each temperature level. The parameters, $\beta_{F}$ and $\beta_{S}$, are the difference rate of the forest cover and the rainfall difference rate, respectively.

The TR terms in Eq.(3.3.1) represent the proportion of rainfall at each temperature level, TS is the proportion of the rainfall difference rate at each temperature level.

The parameter $\pi_{F}$ is the decay rate of the forest caused by water absorption at each temperature level. $\pi_{R}$ And $\pi_{S}$ are the decreasing and increasing rainfall difference rates, respectively, affecting by the proportion of rainfall at each temperature level. The parameters, $\mu_{R}$ and $\mu_{S}$, are the difference rates with which the rainfall changes its behavior at each temperature level.

The RS terms in Eq. (3.3.1) represent the relationship between the rainfall difference rate and the amount of rainfall. Consider the situation where the rainfall amount is near the saturation point; the rainfall difference rate could either be substantially reduced or slightly increased. Conversely, at the low level of rainfall, the rainfall difference rate could either be substantially increased or slightly reduced. The parameters $\delta_{R}$ and $\delta_{S}$ are, respectively, the difference rate of the amount of rainfall and the rainfall difference rate affected by the relationship between the change in the behavior of the rainfall and the amount of rainfall.

\section{Chapter 4 Conclusion}

The relationship between global temperature dynamics, forest area and amounts of rainfall has been mathematically formulated. In this study, there are two models representing the long-term behaviors of rainfall as affected by the rise in global temperature and the decline in the forest cover. The hypotheses for this study are the logistic patterns of global temperature and the amount of forest cover, and the periodic behavior of the rainfall pattern. Since the behavior of rainfall is seasonal the increase in global temperature and decreasing in forest cover cause water management difficult and eventually affect to drought and flood.

In the second part of the study, the model is refined to cover interactions between variables. The parameter $\pi_{S}$ indicates that the rainfall difference rate is affected by the proportion of rainfall at each temperature level. Therefore, global temperature influences the fluctuation of rainfall. The model on the rainfall pattern can further be improved to capture the fluctuations in the annual rainfall amount. To do so, the amplitude of the periodic functions representing the rainfall amount has to fluctuate. It would also be interesting to project the amount of rainfall to the future and to work on some scenario analysis using varying reforestation policies.

\section{Bibliography}

[1] Differential Equations and Boundary value problems. computing and modeling, third edition, Edwards Penney, 2004.

[2] Walter G.Kelley, Allan C.Peterson, 2010. The theory of Differential Equation classical and qualitative, second edition.

[3] Alexiadis, A., 2007. Global warming and human activity: a model for studying the potential instability of the carbon dioxide/temperature feed back mechanism. Ecological Modelling 203, 243-256.

[4] Bampfylde, C.J., Brown, N.D., Gavaghan, D.J., Naini, P.K., 2005. Modeling rain forest diversity: the role of competition. Ecological modeling 188, 253-273.

[5] Borobia, M., Vourch, A., Yunis, E., 2001. Water and tourism Water Policy 3, 1-216.

[6] Caetano, M.A.L., Gherardi, D.F.M., Yoneyama, T., 2008. Optimal resource management control for CO2 emission and reduction of the greenhouse e_ect. Ecological Modelling 213, 119-126.

[7] Dubey, B., Das, B., 1999. Models for the survival of species dependent on resource in industrial environments. Journal of Mathematical Analysis and Applications 231, 374-396. 
[8] Dubey, B., Sharma, S., Sinha, P., Shukla, J.B., 2009. Modelling the depletion of forestry resources by population and population pressure augmented industrialization. Applied Mathematical Modeling 33, 3002-3014. 